\title{
Information and evidence in bargaining
}

\author{
Péter Eső • Chris Wallace
}

Received: 5 September 2013 / Accepted: 20 November 2013 / Published online: 30 November 2013 (C) SAET 2014

\begin{abstract}
In a simple bilateral bargaining model with two-sided private information, players may receive signals about the value of the available surplus. The equilibrium of this game when signals are unverifiable information is qualitatively different from when they constitute verifiable evidence: in the latter case some proposer types will always separate, whereas in the former no information is ever transmitted. Despite the presence of "unraveling", the proposer is always better off with verifiable evidence, although efficiency may be adversely affected. Interpreting the ability to generate verifiable evidence as bargaining skill, a player benefits from being more skilful and may also be better off with a more skilful rival.
\end{abstract}

Keywords Bargaining - Two-sided private information - Unverifiable information . Verifiable evidence

JEL Classification $\quad \mathrm{C} 78 \cdot \mathrm{D} 82 \cdot \mathrm{D} 83$

\section{Unverifiable information vs. verifiable evidence}

This paper investigates the effect that the verifiability of information has upon the equilibrium strategies and payoffs in a simple bargaining game. The context is a bilateral trade setting with two-sided private information about the size of the potential surplus. A proposer $(S)$ makes a take-it-or-leave-it offer to the other player $(B)$. At

\footnotetext{
P. Eső (ه)

Department of Economics, University of Oxford, Oxford OX1 3UQ, UK

e-mail: peter.eso@economics.ox.ac.uk

C. Wallace

Department of Economics, University of Leicester, Leicester LE1 7RH, UK

e-mail: cw255@leicester.ac.uk
} 
the point when this offer is made both parties may have received private signals about the available surplus. The paper compares the game's equilibrium when those signals are unverifiable with the situation when the signals are fully and freely verifiable. (In both cases, the absence of information cannot be proven.)

When information is unverifiable the equilibrium involves pooling: $S$ cannot credibly separate to reveal information received by using different take-it-or-leave-it offers. Depending on whether the other player, $B$, is likely to be informed or not, the pooling offer is either "high" or "medium". In contrast, when evidence is verifiable, a player who receives favourable information discloses it and thereby convinces the other to agree to a particular offer. Hence, when $S$ discloses no information, $B$ becomes more pessimistic about the value of the surplus and accepts only a relatively "low" offer (this is the "unraveling" effect). ${ }^{1}$ The equilibrium in this case may involve full separation of $S$ 's types depending on the players' abilities to obtain information.

The proposer is always better off ex ante in the verifiable information regime. The fact that offers must be low when evidence is verifiable and no disclosure is made is more than offset in equilibrium by the chance that a favourable piece of evidence becomes available regarding the value of agreement. This is true even though the expected size of the surplus generated may fall when moving from unverifiable information to verifiable evidence, resulting from an inefficient lack of agreement.

Moreover, the bargaining framework studied here allows questions to be answered concerning the players' "bargaining skill", interpreted as their ability to generate verifiable evidence. It is always beneficial for the proposer $S$ to be better informed when the evidence is verifiable. Depending on the parameters, a more highly skilled rival may increase or decrease a player's ex-ante payoff. On the other hand, efficiency is always (weakly) enhanced by an increase in the players' bargaining skills.

In the literature on dynamic bargaining games following Rubinstein (1982), private information, if modeled, is typically assumed to be unverifiable. ${ }^{2}$ Information structures with (partially) verifiable evidence have been applied mainly in informationtransmission (persuasion) games rather than in bargaining (Milgrom 2008, provides a survey). One nice exception is the model of Shavell (1994) who studies a rather different trading game with information disclosure where the focus is on the (in)efficiency of information acquisition rather than on the effects of verifiability itself. Thus this paper represents a first step towards understanding the effects of the verifiability of information in bargaining.

\section{The bargaining game}

Two players $B$ (she, a buyer perhaps) and $S$ (he, a seller) bargain over a potential surplus $v \in\{0,1\}$, where $\operatorname{Pr}[v=1]=\sigma \in(0,1)$ is the common prior that there are positive gains from agreement. If agreement is reached, the surplus (if any) is realized by $B$.

\footnotetext{
1 Milgrom (1981) and Grossman (1981) were the first to identify this effect in persuasion games. Shin (1994), Seidmann and Winter (1997), and Mathis (2008) study information-transmission games with this feature.

2 Kennan and Wilson (1993), or more recently Ausubel et al. (2002), provide surveys.
} 
$B$ and $S$ independently and privately learn $v$ with probabilities $r_{B}$ and $r_{S}$, respectively. Writing $b$ and $s$ for the players' respective signals, then

$$
\operatorname{Pr}[b=v]=1-\operatorname{Pr}[b=\emptyset]=r_{B} \quad \text { and } \quad \operatorname{Pr}[s=v]=1-\operatorname{Pr}[s=\emptyset]=r_{S},
$$

where $r_{B}, r_{S} \in(0,1)$ and $\emptyset$ denotes the empty signal realization where $v$ is not observed. An informative signal is fully revealing: once $v$ has been observed by a player, no further (non-trivial) updating takes place by that player.

The signals received by $B$ and $S$ may be unverifiable information or verifiable evidence. In the former case, neither $B$ nor $S$ can make any verifiable disclosure to the other player. In the latter case, $B$ and $S$ have a simultaneous opportunity to credibly and verifiably disclose $b=v$ or $s=v$; if any such disclosure takes place, $v$ immediately becomes common knowledge to the players. Neither $B$ nor $S$ can disclose an empty signal, $\varnothing$, however.

After the signals are realized and whether or not disclosure has taken place, $S$ makes a take-it-or-leave-it offer $p$ to $B$ in exchange for agreement. If accepted, $B$ must pay $S$ the price $p$ and payoffs are given by $v-p$ for $B$ and $p$ for $S$. If rejected, the game ends with payoffs of zero to both players.

Players strictly prefer agreement with zero payoff to failing to agree. ${ }^{3}$ Lexicographic preference for agreement when payoffs are zero eliminates equilibria relying on $B$ 's indifference between accepting and rejecting; this simplifies the analysis without altering the model's essential features. This assumption has particular relevance when information is unverifiable. Its significance in this case is discussed in more depth following the equilibrium characterization of Proposition $1 . B_{b}$ is the notation for the $B$ type who observed a signal $b \in\{0,1, \emptyset\}$. $S_{S}$ is analogous notation for the $S$ type who received $s \in\{0,1, \emptyset\}$.

\section{Equilibrium characterization and payoffs}

This section provides a characterization of perfect Bayesian equilibria (PBE) of the game that are uniformly preferred by all types of proposers ( $S$-optimal). This refinement eliminates all PBE that yield weakly lower payoffs to every $S$ type and a strictly lower payoff to at least one type of $S$. Since players are assumed strictly to prefer agreement with a zero payoff to disagreement, all $S$-optimal PBE will be in pure strategies. Moreover, in the game studied here, there exists a unique $S$-optimal PBE. ${ }^{4,5}$

\footnotetext{
3 Non-pecuniary benefits generated by partnership formation or by trading could motivate this assumption.

4 Formally, it can be shown that the perfect sequential equilibrium (PSE) refinement introduced by Grossman and Perry (1986) would eliminate all PBE but the $S$-optimal one. With unverifiable information the PBE are all pooling, and the PSE refinement eliminates those with uniformly lower payoffs to each $S$ type; with verifiable evidence, the equilibria yielding lower payoffs to the $S$ types who pool are eliminated.

5 A further rationale for the selection of this equilibrium could be provided by a forward-induction argument: if there were a previous period in which $B$ made an offer to $S$ (in the spirit of an alternating-offer bargaining game), $S$ could indicate that he wants to play the equilibrium most preferred by all of his types by rejecting any offer lower than the continuation payoff he would receive from the $S$-optimal equilibrium.
} 
When verifiable evidence is available, it is dominant for $S_{1}$ to disclose $s=1$ and set $p=1$. $B$ will accept this (as it yields a zero payoff, and acceptance is strictly preferred in such instances by assumption). $B_{0}$ will likewise disclose $b=0$, ensuring $p=0$ is offered by $S$ which $B_{0}$ can accept. However, when only unverifiable information is available, $S_{1}$ may find it impossible to separate. Consequently, the sets of equilibria under the two regimes will be different and are treated separately.

\subsection{Unverifiable information}

First, consider the information regime when signals may not be disclosed verifiably to the other party. In this circumstance, as the following proposition demonstrates, $S_{1}$ cannot credibly separate from the other two types of $S$.

Proposition 1 With unverifiable information the unique S-optimal PBE strategy profile satisfies:

(i) If $r_{B} \leq \sigma$, then all types of $S$ pool on an offer of $p=\sigma$, which $B_{1}$ and $B_{\emptyset}$ accept; any other offer is rejected by all types except $B_{1}$. The ex-ante payoff to $S$ is $\left[1-r_{B}(1-\sigma)\right] \sigma$. Expected surplus is maximized: if $v=1$, agreement is reached with probability 1 .

(ii) If $r_{B}>\sigma$, then $S_{1}$ and $S_{\emptyset}$ pool on $p=1$, which $B_{1}$ alone accepts. $S_{0}$ offers $p=0$, which all types of $B$ accept. The ex-ante payoff to $S$ is $r_{B} \sigma$. Bargaining is inefficient: if $v=1$, agreement is reached only if $b=1$ and thus expected surplus is $r_{B} \sigma$.

Proof First, consider equilibria in which $S_{0}$ separates from the other two types. In any such equilibrium $S_{0}$ must set $p\left(S_{0}\right)=0$, to be accepted by $B_{\emptyset}$ and $B_{0}$, yielding zero monetary payoff. Any other (positive) price set by $S_{\emptyset}$ or $S_{1}$ may be accepted only by $B_{1}$ (a type of $B$ considered to be impossible by $S_{0}$ ), because otherwise $S_{0}$ would have an incentive to deviate. Therefore, both $S_{1}$ and $S_{\emptyset}$ must offer the same $\bar{p}$, to be accepted by $B_{1}$ alone; the $S$-optimal PBE in this class has $\bar{p}=1$. The PBE can be sustained by $B_{\emptyset}$ believing that any deviation to $p \in(0,1)$ comes from $S_{0}$ and therefore rejecting it. $S$ 's expected payoff in this case is the probability that $v=1$ and $B$ observes it, $r_{B} \sigma$, which is also the expected surplus. $S$ extracts all the surplus since $p=1$ whenever agreement is reached at positive $p$.

Second, consider equilibria in which all types of $S$ pool on setting the same $\bar{p}>0$. If only $B_{1}$ accepts $\bar{p}$, then $S_{0}$ gets zero monetary payoff with no agreement: he would prefer to set $p=0$, which would be accepted by both $B_{\emptyset}$ and $B_{0}$, leading to a contradiction. Hence the pooling price $\bar{p}>0$ must be accepted by both $B_{\emptyset}$ and $B_{1}$, which implies $\bar{p} \leq \sigma$. The pooling equilibrium exists as long as neither $S_{1}$ nor $S_{\emptyset}$ prefers to deviate to $p=1$ to be accepted by $B_{1}$. $S_{1}$ believes he is facing $B_{1}$ with probability $r_{B}$, whereas $S_{\emptyset}$ is less optimistic. Therefore, the PBE in which all types of $S$ pool on $\bar{p}>0$ exists as long as $r_{B} \leq \bar{p} \leq \sigma$. The $S$-optimal PBE in this class has $\bar{p}=\sigma$ and exists under the least restrictive condition, $r_{B} \leq \sigma$. Clearly, when this type of equilibrium exists, it is preferred by all types of $S$ to the one in which $S_{1}$ and $S_{\emptyset}$ pool on $\bar{p}=1$ (accepted only by $B_{1}$ ). This follows from the conditions of existence 
and that $S_{0}$ makes a positive expected payoff. In this equilibrium agreement (at price $\sigma$ ) is reached so long as $B$ does not observe $v=0$ : S's ex-ante payoff is therefore $\left[1-r_{B}(1-\sigma)\right] \sigma$, and expected surplus is maximized.

In any other equilibrium, either $S_{0}$ pools with $S_{\emptyset}$ (that is, $S_{1}$ separates), or $S_{0}$ pools with $S_{1}$ (that is, $S_{\emptyset}$ separates). The proof that no such equilibria exist proceeds by contradiction.

In the former case, $S_{1}$ would set $p\left(S_{1}\right)=1$, accepted by both $B_{1}$ and $B_{\emptyset}$. Suppose $S_{0}$ and $S_{\emptyset}$ pool on $\hat{p}$. If $\hat{p}=0$, then $S_{0}$ would find it optimal to imitate $S_{1}$ (to get a strictly positive expected payoff). If $\hat{p} \in(0,1)$ then $S_{0}$ considers it acceptable only to $B_{\emptyset}$ at most; but then $p\left(S_{1}\right)=1$ would be accepted with at least the same probability and generate a higher expected payoff for $S_{0}$ : again, $S_{0}$ would wish to imitate $S_{1}$. Therefore, no such PBE exists.

In the latter case $\left(S_{1}\right.$ and $S_{0}$ pool on $\hat{p}>0$ and $S_{\emptyset}$ separates), price $\hat{p}$ would have to be accepted by both $B_{1}$ and $B_{\emptyset}$, for if only $B_{1}$ accepted then $S_{0}$ would prefer to deviate [either to $p\left(S_{\emptyset}\right)$ or to $p=0$ ]. Therefore, $p\left(S_{\emptyset}\right) \neq \hat{p}$ must be accepted by $B_{1}$ alone (otherwise $S_{1}$ and $S_{\emptyset}$ would both set whichever price is highest of the two). Type $S_{1}$ has no incentive to deviate if $\hat{p} \geq r_{B} p\left(S_{\emptyset}\right)$, whereas the incentive constraint for $S_{\emptyset}$ is $\hat{p} \leq \mu p\left(S_{\emptyset}\right)$, where

$$
\mu=\operatorname{Pr}[b=1 \mid s=\emptyset, b \in\{\emptyset, 1\}]=\frac{r_{B} \sigma}{1-r_{B}(1-\sigma)} .
$$

These yield a contradiction because $\mu<r_{B}$, which completes the proof.

It should not altogether be surprising that $S_{1}$ cannot separate when information is unverifiable. Separation would entail $B_{\emptyset}$ learning that $v=1$ for sure when $p=1$, which, under the assumption that players strictly prefer agreement when payoffs are zero, would require her to accept. As a result $S_{0}$ would wish also to set $p\left(S_{0}\right)=1$, a contradiction. Only if $B_{\emptyset}$ accepted $p=1$ with a low enough probability to deter such a deviation by $S_{0}$ could an equilibrium of this kind be sustained; this is ruled out by the preference structure. ${ }^{6}$ In addition, such mixed equilibria would not be particularly compelling. They would involve $B_{\emptyset}$ mixing only to improve $S$ 's payoffs: something she has no incentive to do.

\subsection{Verifiable evidence}

When the signals are verifiable, $S_{1}$ will disclose $s=1$ and $B_{0}$ will disclose $b=0$. Both disclosures result in immediate agreement with $p=v$. Thus, if $S$ does not disclose $s=1, B_{\emptyset}$ must update her beliefs to

\footnotetext{
${ }^{6}$ Without the lexicographic preference structure, mixed equilibria do indeed exist for certain parameter values. For instance, there are equilibria where $S_{1}$ sets $p=p^{H}>\sigma, S_{0}$ sets $p=p^{L}<\sigma$, and $S_{\emptyset}$ mixes over (possibly a subset of) $\left\{p^{L}, \sigma, p^{H}\right\} . B_{1}$ accepts any $p \in\left\{p^{L}, \sigma, p^{H}\right\}$, whereas $B_{\emptyset}$, made indifferent to accepting or rejecting any of the equilibrium prices by $S_{\emptyset}$ 's mixing, rejects $p^{H}$ and $\sigma$ with positive probabilities ( $p^{H}$ more often than $\sigma$ ). Mixing by $B_{\emptyset}$ then makes $S_{\emptyset}$ indifferent among the prices he offers with positive probability. There could also be pure equilibria with the (trivial) difference that $B_{0}$ rejects all offers.
} 


$$
\beta=\operatorname{Pr}[v=1 \mid b=\emptyset, s \in\{0, \emptyset\}]=\frac{\left(1-r_{S}\right) \sigma}{1-r_{S} \sigma} .
$$

In the unique $S$-optimal PBE when neither $S$ has disclosed $s=1$ nor $B$ has disclosed $b=0$, the remaining $S$ types will either pool on $p\left(S_{0}\right)=p\left(S_{\emptyset}\right)=\beta$ (accepted by all types of $B$ ) or they will separate with $p\left(S_{0}\right)=0$ (accepted for sure) and $p\left(S_{\emptyset}\right)=1$ (accepted by $B_{1}$ only). Which of these two cases applies depends on which of the quantities defined in Eqs. (1) and (2) is the larger, that is whether or not $\mu \leq \beta$. Now,

$$
\mu \leq \beta \Leftrightarrow r_{B}(1-\sigma) \leq\left(1-r_{B}\right)\left(1-r_{S}\right)
$$

This is equivalent to $r_{B} \leq\left(1-r_{S}\right) /\left(1-r_{S}+1-\sigma\right) \equiv R_{B}\left(r_{S}\right){ }^{7}$ The next proposition characterizes the equilibrium in the presence of verifiable evidence.

Proposition 2 With verifiable evidence, the unique S-optimal PBE strategy profile satisfies:

(i) If $\mu \leq \beta$, then $S_{1}$ discloses $s=1$ and offers $p\left(S_{1}\right)=1$ (which is accepted for sure), $B_{0}$ discloses $b=0$ forcing $S_{\emptyset}$ and $S_{0}$ to offer $p=0$ (which $B_{0}$ accepts), and if $b \neq 0, S_{\emptyset}$ and $S_{0}$ pool on an offer of $p=\beta$ which either type of $B$ ( $B_{1}$ and $\left.B_{\emptyset}\right)$ accepts. The ex-ante payoff to $S$ is $\sigma-r_{B}(1-\sigma) \beta$. The expected surplus is maximized: if $v=1$, agreement is reached with probability 1 .

(ii) If $\mu>\beta$, then $S_{1}$ discloses $s=1$ and offers $p\left(S_{1}\right)=1$ (which is accepted for sure), $B_{0}$ discloses $b=0$ forcing $S_{\emptyset}$ and $S_{0}$ to offer $p=0$ (which $B_{0}$ accepts), and if $b \neq 0, S_{\emptyset}$ offers $p\left(S_{\emptyset}\right)=1$ (which $B_{1}$ alone accepts) and $S_{0}$ offers $p\left(S_{0}\right)=0$, which $B_{\emptyset}$ accepts. The ex-ante payoff to $S$ is $\left[r_{S}+\left(1-r_{S}\right) r_{B}\right] \sigma$. Bargaining is inefficient: if $v=1$, agreement is not reached when $s=b=\emptyset$ and thus the expected surplus is $\left[1-\left(1-r_{B}\right)\left(1-r_{S}\right)\right] \sigma=\left[r_{S}+\left(1-r_{S}\right) r_{B}\right] \sigma$.

Proof If $S_{\emptyset}$ 's equilibrium strategy prescribes setting a price that is accepted by $B_{\emptyset}$ then $S_{0}$ sets the same $p$ (otherwise he foregoes a positive payoff); therefore $p$ conveys no further information and so $p \leq \beta$. In the $S$-optimal PBE, therefore, $p=\beta$.

If $S_{\emptyset}$ 's equilibrium strategy prescribes setting a price that is accepted only by $B_{1}$, then $S_{0}$ gets zero payoff for sure, and hence sets $p\left(S_{0}\right)=0$ which $B_{\emptyset}$ accepts (knowing that $v=0)$. In this case the $S$-optimal PBE must involve $p\left(S_{\emptyset}\right)=1$.

In the former case, $S_{\emptyset}$ believes $p=\beta$ is accepted for sure, since $B_{0}$ would have disclosed $b=0$. In the latter case $S_{\emptyset}$ believes that, again since $b \neq 0, p\left(S_{\emptyset}\right)=1$ is accepted with probability $\operatorname{Pr}[b=1 \mid s=\emptyset, b \neq 0]=\mu$, defined in Eq. (1). Hence, the former case (i) applies whenever $\mu<\beta$ and the latter case (ii) whenever $\mu>\beta$. Note further that no pooling equilibrium can exist when $\mu>\beta$. Were $S_{\emptyset}$ and $S_{0}$ to pool, the best price at which to do so is $\beta$ (accepted with certainty). But now, $S_{\emptyset}$ would be strictly better off deviating to a price of 1 , accepted (by $B_{1}$ alone) with probability $\mu$.

When $\mu=\beta$ exactly, $S_{\emptyset}$ is precisely indifferent between the two cases, but $S_{0}$ would strictly prefer to offer a price of $\beta$. Thus, case (i) applies if $\mu=\beta$ in the $S$-optimal PBE.

\footnotetext{
${ }^{7}$ Figure 1 illustrates this inequality: the area under the $\mu=\beta$ line (labeled $\mathrm{A}$ and $\mathrm{C}$ ) corresponds to $\mu<\beta$.
} 
In case (i), agreement is reached when $v=1$ and $S$ observes it (at $p=1$ ), when $v=$ 1 but $S$ does not observe it (at price $\beta$ ), or when $v=0$ but $B$ does not observe it (again at price $\beta)$. Therefore the ex-ante payoff to $S$ is $r_{S} \sigma+\left[\left(1-r_{S}\right) \sigma+\left(1-r_{B}\right)(1-\sigma)\right] \beta$, which can be rewritten as $\sigma-r_{B}(1-\sigma) \beta$. In case (ii), agreement takes place (with $p=1$ ) only if $v=1$ and either $S$ observes it or, if not, $B$ does. $S$ 's ex-ante payoff is therefore $\left[r_{S}+\left(1-r_{S}\right) r_{B}\right] \sigma$.

\subsection{Payoff comparison of information regimes}

Note that, from Proposition 1, the ex-ante equilibrium payoff to $S$ does not depend upon his ability to generate information about $v$. This is intuitive: information is of no value to $S$ when it cannot be used to credibly convince $B$ of the size of the surplus.

However, from Proposition 2, when evidence is verifiable, $S$ 's ex-ante payoff is increasing $r_{S} . S$ is better off when he is better able to generate information. This is not as obvious as it may first appear: being known to be more able to identify $v$ reduces the price that $B_{\emptyset}$ is willing to accept in the event that $S$ cannot disclose $s=1$. This is precisely the unraveling effect alluded to earlier. Nevertheless, ex ante, $S$ is always better off with a larger $r_{S}$.

Loosely speaking, being totally uninformed $\left(r_{S} \rightarrow 0\right)$ when evidence is verifiable leaves $S$ in exactly the same position as when information is unverifiable. Increasing $r_{S}$ benefits $S$ in the former case, but not in the latter. As a result, $S$ is always going to be better off with verifiable evidence for $r_{S} \in(0,1)$. The next proposition formalizes this intuition and also provides a comparison of the efficiency properties of the two regimes.

Proposition 3 For any $\left(r_{B}, r_{S}, \sigma\right) \in(0,1)^{3}$ the ex-ante payoff for $S$ in the $S$-optimal $P B E$ is strictly smaller with unverifiable information than it is with verifiable evidence. Expected surplus is strictly larger with unverifiable information than it is with verifiable evidence when $r_{B} \leq \sigma$ and $\mu>\beta$, strictly smaller when $r_{B}>\sigma$, and otherwise the same.

Proof There are four cases to consider, corresponding to the regions A, B, C, and D in Figure 1. First, consider area A. This corresponds to $r_{B} \leq \sigma$ and $\mu \leq \beta$, or case (i) in Propositions 1 and 2. Now $\left[1-r_{B}(1-\sigma)\right] \sigma<\sigma-r_{B}(1-\sigma) \beta$ since $\beta<\sigma<1$. Moreover, in these cases if $v=1$, agreement is reached with certainty, so the expected surplus is the same.

In area $\mathrm{B}$ where $r_{B} \leq \sigma$ and $\mu>\beta$, or cases (i) in Proposition 1 and (ii) in Proposition 2, the claim is $\left[1-r_{B}(1-\sigma)\right] \sigma<\left[r_{S}+\left(1-r_{S}\right) r_{B}\right] \sigma$. This can be rewritten as $\left(1-r_{B}\right)\left(1-r_{S}\right)<r_{B}(1-\sigma)$ which is true when $\mu>\beta$ from the equivalence given in Eq. (3). If $v=1$, agreement is reached with certainty with unverifiable information, but with probability $1-\left(1-r_{B}\right)\left(1-r_{S}\right)<1$ with verifiable evidence.

In area $\mathrm{C}$ where $r_{B}>\sigma$ and $\mu \leq \beta$, or cases (ii) in Proposition 1 and (i) in Proposition 2, the claim is $r_{B} \sigma<\sigma-r_{B}(1-\sigma) \beta$ or equivalently $r_{B}(1-\sigma) \beta<$ $\left(1-r_{B}\right) \sigma$. Again, using Eq. (3), $\mu \leq \beta$ is equivalent to $r_{B}(1-\sigma) \beta \leq\left(1-r_{B}\right)\left(1-r_{S}\right) \beta$, which is indeed less than $\left(1-r_{B}\right) \sigma$ because $\beta<\sigma$ and $r_{S}>0$, confirming the claim. 


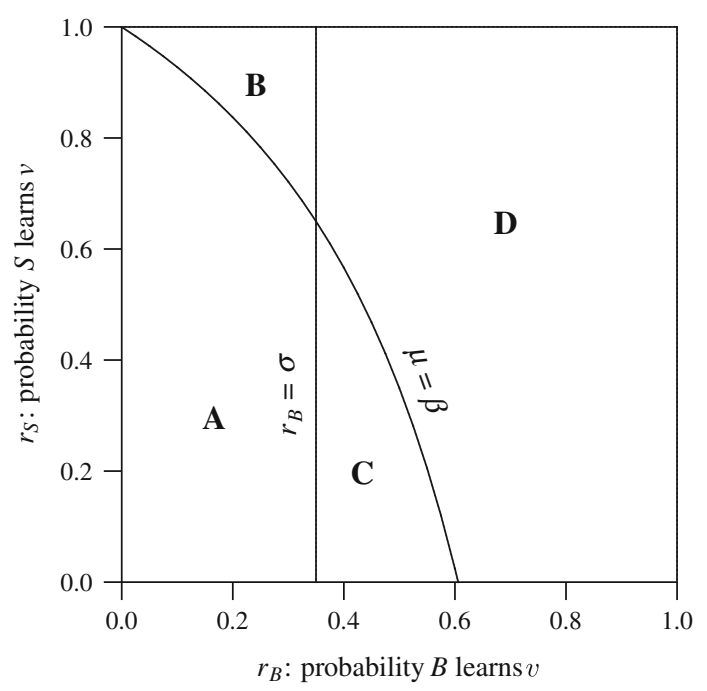

Fig. $1 S$-optimal perfect Bayesian equilibrium. The figure illustrates the parameter regions associated with the different cases of equilibrium for both unverifiable information and verifiable evidence (with $\sigma=0.35$ ). Areas A and B correspond to case (i) in Proposition 1: $r_{B} \leq \sigma$ and $S$ pools on $p=\sigma$. In areas C and $\mathrm{D}, r_{B}>\sigma$ and $S$ pools on $p=1$ : case (ii) in Proposition 1. In areas A and C case (i) of Proposition 2 applies, since $\mu \leq \beta$, so $S_{\emptyset}$ and $S_{0}$ pool on $p=\beta$, whereas in areas B and D, $\mu>\beta$ and so case (ii) of Proposition 1 applies: $S$ fully separates ( $S_{\varnothing}$ offers 1 and $S_{0}$ offers 0 )

If $v=1$, agreement occurs with probability $r_{B}$ with unverifiable information, but for sure with verifiable evidence.

Finally, in area D where $r_{B}>\sigma$ and $\mu>\beta$, or case (ii) in Propositions 1 and 2, the claim is $r_{B} \sigma<\left[r_{S}+\left(1-r_{S}\right) r_{B}\right] \sigma$. This is clearly true since $r_{B}, r_{S} \in(0,1)$. If $v=1$, agreement is reached with probability $r_{B}$ when information is unverifiable, but with probability $1-\left(1-r_{B}\right)\left(1-r_{S}\right)>r_{B}$ when evidence is verifiable.

Comparing the fortunes of the three types of $S$ in the various areas identified in Fig. 1 is instructive. Consider first areas $C$ and D. Here, all types of $S$ are weakly better off with verifiable evidence: in C, $S_{0}$ is now able to make a positive offer, accepted by $B_{\emptyset}$; $S_{1}$ continues to offer 1 , but this is now accepted by $B_{\emptyset}$ as well as $B_{1} ; S_{\emptyset}$ now makes a lower offer, but it is accepted more often which more than compensates. In area D things are even more straightforward. All $S$ types make the same offers as under unverifiable information, but $S_{1}$ is able to obtain agreement from $B_{\emptyset}$ as well as $B_{1}$.

Areas A and B illustrate the potential problem for $S$ posed by verifiable information. Although $S_{1}$ is strictly better off being able to reveal credibly $s=1$, in A both $S_{\varnothing}$ and $S_{0}$ must now make lower offers $(\beta<\sigma)$ without any increase in the chances of agreement. Similarly in B, $S_{0}$ must lower his price (to zero, and thus is certainly worse off) and $S_{\emptyset}$ must raise his price (to 1 , which is accepted by $B_{1}$ alone, making him worse off). Nevertheless, as Proposition 3 demonstrates, ex-ante payoffs to $S$ are higher with verifiable information: the bonus associated with convincing $B_{\emptyset}$ that $v=1$ when $s=1$ always outweighs the impact of reduced offers via the unraveling effect when $s \neq 1$. 


\section{Bargaining skill and equilibrium payoffs}

The relationship between $S$ 's ex-ante payoff and $r_{S}$ has already been discussed in Sect. 3. However, the relationship between his payoff and $r_{B}$ is slightly more involved: fixing all else, $S$ 's payoff is first decreasing and then increasing in $r_{B}$. In both Propositions 1 and 2 case (i) applies when $r_{B}$ is low and case (ii) when $r_{B}$ is high, but the condition for which case applies is different in the two information regimes. As a consequence, a change in $r_{B}$ can have perverse effects upon $S$ 's equilibrium payoff across the two regimes. Surplus, on the other hand, behaves in an intuitive manner: it is everywhere increasing (albeit weakly) in bargaining skill. These comparative static results are summarized in the final proposition. ${ }^{8}$

Proposition 4 The expected payoff for $S$ in the $S$-optimal PBE is invariant to $r_{S}$ with unverifiable information, but it is increasing in $r_{S}$ with verifiable evidence. The expected payoff for $S$ is increasing in $r_{B}$ with unverifiable information and decreasing in $r_{B}$ with verifiable evidence when $r_{B} \in\left[\sigma, R_{B}\left(r_{S}\right)\right]$ for $r_{S}<1-\sigma$, whereas it is decreasing in $r_{B}$ with unverifiable information and increasing in $r_{B}$ with verifiable evidence when $r_{B} \in\left[R_{B}\left(r_{S}\right), \sigma\right]$ for $r_{S}>1-\sigma$. Expected surplus is (weakly) increasing in $r_{B}$ and $r_{S}$.

Consider, for instance, the case when $r_{S}$ is high and evidence is verifiable. In this case $S_{\emptyset}$ is being "forced" to offer $p=1$ because to ensure acceptance by $B_{\emptyset}$ would require a very low offer: $B$ has radically updated her belief that $v=1$ when $S$ failed to disclose ( $r_{S}$ is high, so from $B$ 's perspective it is now very probable that he is concealing $s=0$ ). Hence, if $B$ were better informed (better skilled at generating verifiable evidence), agreement would occur more often in the event $v=1$, benefiting $S$. In contrast, when information is unverifiable $S$ pools on $p=\sigma$ when $r_{B}$ is low (and this is accepted by $B_{\emptyset}$ ); a higher $r_{B}$ increases the probability of rejection by raising the chances that $b=0$, which hurts $S$.

Acknowledgments The authors would like to thank the editor and an anonymous referee for helpful comments and advice.

\section{References}

Ausubel, L.M., Cramton, P., Deneckere, R.J.: Bargaining with incomplete information. In: Aumann, R.J., Hart, S. (eds.) Handbook of Game Theory with Economic Applications, chap. 50, vol. III, pp. 1897-1945. Elsevier, Amsterdam (2002)

Grossman, S.J.: The informational role of warranties and private disclosure about product quality. J. Law Econ. 24(3), 461-483 (1981)

Grossman, S.J., Perry, M.: Perfect sequential equilibrium. J. Econ. Theory 39(1), 97-119 (1986)

Kennan, J., Wilson, R.: Bargaining with private information. J. Econ. Lit. 31(1), 45-104 (1993)

Mathis, J.: Full revelation of information in sender-receiver games of persuasion. J. Econ. Theory 143(1), 571-584 (2008)

Milgrom, P.R.: Good news and bad news. Bell J. Econ. 12(2), 380-391 (1981)

Milgrom, P.R.: What the seller won't tell you: persuasion and disclosure in markets. J. Econ. Perspect. 22(2), 115-131 (2008)

\footnotetext{
8 The proof is by straightforward differentiation of the quantities in Propositions 1 and 2, and so is omitted.
} 
Rubinstein, A.: Perfect equilibrium in a bargaining model. Econometrica 50(1), 97-109 (1982)

Seidmann, D.J., Winter, E.: Strategic information transmission with verifiable messages. Econometrica 65(1), 163-169 (1997)

Shavell, S.M.: Acquisition and disclosure of information prior to sale. RAND J. Econ. 25(1), 20-36 (1994)

Shin, H.S.: The burden of proof in a game of persuasion. J. Econ. Theory 64(1), 253-264 (1994) 